\title{
Successful Re-implantation of Intrathecal Delivery System after Removal Secondary to Infection or Wound Dehiscence
}

\author{
Yinn Cher Ooi, BS', Jennifer Malone MSN, CRNP², Teresita DeVera BSN, RN, \\ CNRN $^{2}$, Carol Blyzniuk, BSN, RN², Ashwini Sharan, MD \\ 1Jefferson Medical College, Thomas Jefferson University, Philadelphia, PA \\ 2Department of Neurological Surgery, Jefferson University Physicians, Philadelphia, PA \\ ${ }^{3}$ Department of Neurological Surgery, Thomas Jefferson University, Philadelphia, PA
}

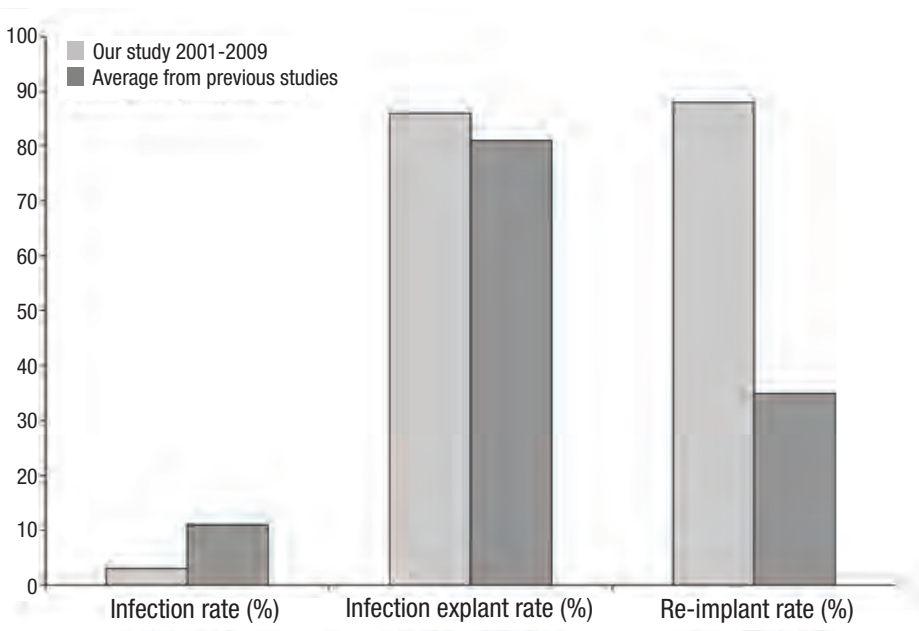

Figure 1

Comparison of our results to previous studies.

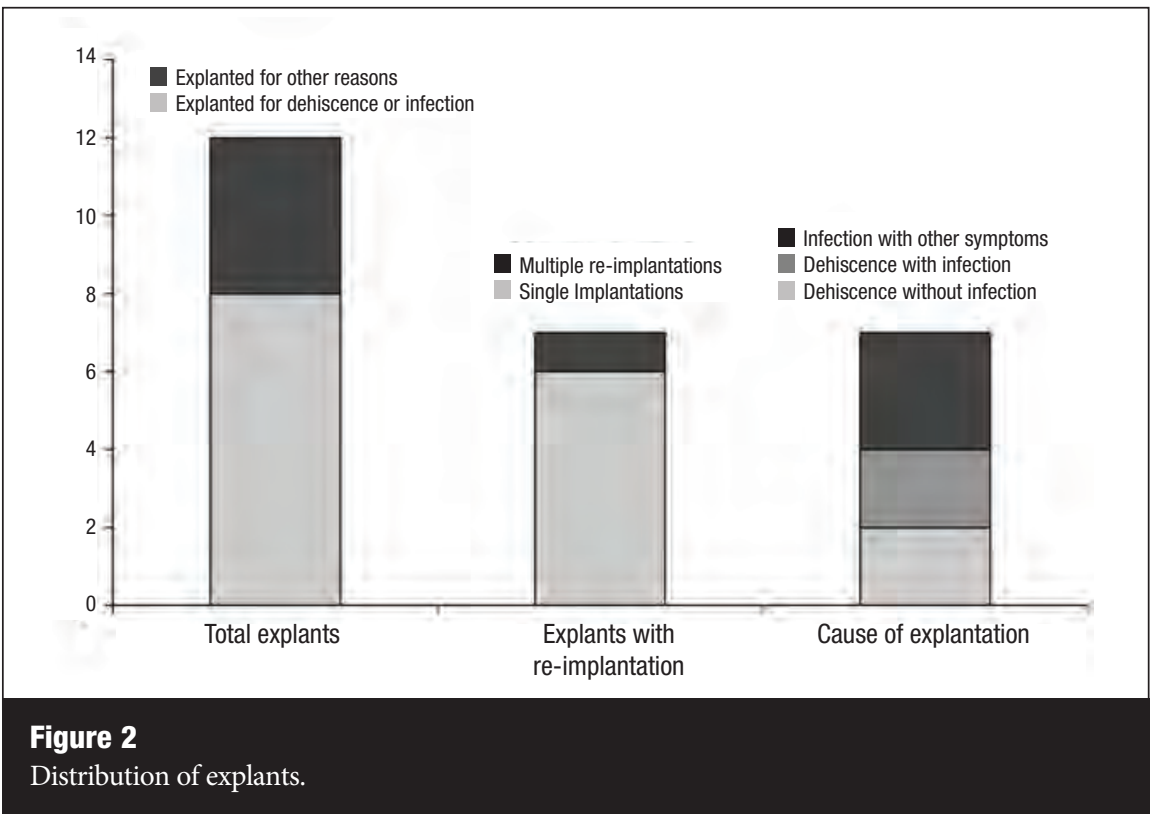

An intrathecal delivery system allows direct infusion of analgesics and antispasmodic drugs into the cerebral spinal fluid in patients with chronic intractable pain or spasticity. Intrathecal therapy effective but any surgical intervention carries the risk of complications. Complications encountered with this therapy include wound dehiscence (spontaneous reopening) and infection, which often lead to explanation of the intrathecal pump. Later re-implanation is feasible, but there is limited information regarding successful retention of the device after re-implantation. A recurring problem with generating reliable guidelines in neurosurgery is that some situations occur too rarely for evidence to be much more than anecdotal.

We wanted to establish a protocol for patient selection for re-implantation prior to surgery, using data acquired at our own institution. Between 2001 and 2009, a total of 219 implant procedures, including revisions, wereperformed in 175 patients. Of these, 12 patients had their intrathecal system explanted. Comparing our results to pooled data from prior publications (Figure 1), we have a lower infection rate and a higher re-implantation rate.

Figure 2 shows the breakdown of explants and re-implantations. 8/12 explants were secondary to an infection and/or wound dehiscence. Seven of the explanted patients were subsequently re-implanted. The average period between explantation and re-implantation was 13 months (range 1-26 months) and re-implanted patients were followed up for a mean period of 26 months (range 4-51 months).

No specific co-morbidity was predictive of explantation. Of the seven re-implanted patients, six had a single episode of infection or dehiscence leading to explantation and one had three episodes of either dehiscence or infection leading to multiple explantations and re-implantations. The multiple complications experienced by that single patient were attributed to a congenital connective tissue and lymphatic system disorder which created a special challenge for wound healing. Three patients, including the patient with multiple reimplantations, had their system explanted secondary to infection at the pump site with noted 


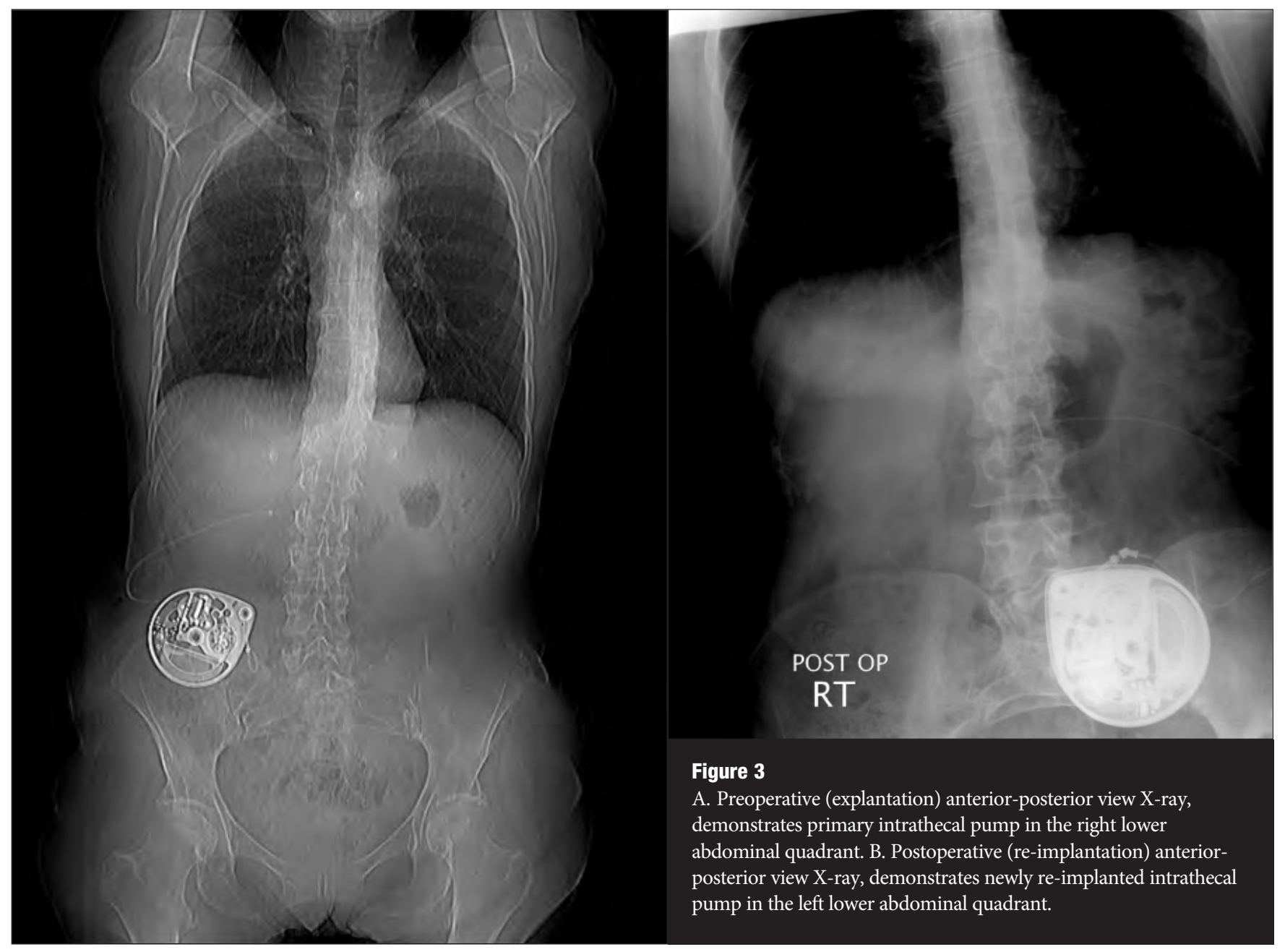

symptoms of erythema, edema, drainage and tenderness; two were explanted for incisional dehiscence without clinical sign of infection; and two patients had incisional dehiscence along with clinical signs of infection.

In all seven, the pump re-implantations took place with no complications, including no recurrence of infections, pump contamination or incisional dehiscence. Since our practice represents a small sample, conclusions are difficult to generalize. Re-implantation was performed at a new/distant site (opposite side of the abdomen or buttocks). An example is shown in Figure 3. All explanted systems were replaced by new intrathecal pumps during re-implantation. All re-implanted intrathecal systems were functioning at optimal levels, providing adequate symptomatic relief.

Similarities were found among the seven re-implanted patients, which may explain how they have successfully retained their re- implanted devices without additional infection or dehiscence. All patients completed a course of antibiotics and remained infection-free prior to re-implantation. All patients lived in a private family dwelling, and were therefore at a lower risk for exposure to iatrogenic infections. All patients had access to assistance in the home if needed for activities of daily living (ADLs), including repositioning (for example, getting into and out of bed) and mobility around the house, and instrumental activities of daily living (IADLs) which include light housework, preparing meals, and shopping for groceries or clothes. All patients had convenient access to health care providers. Only one of the seven patients used tobacco. Six of the seven patients were noted to have adequate nutritional status.

Using this data, a protocol can be created to ensure the success of future patients who require explanation of their baclofen pump due to dehiscence or infection:
- Have patients completed a course of antibiotics and remained infection free after a period of time off all antibiotics?

- Have serum infection and nutrition markers been measured at set point intervals?

- Has the patient ceased all tobacco products?

- Is there support or assistance for ADLs/IADLs, including mobility and repositioning?

Further, a more systematic approach to patient education during the time between explantation and re-implantation should be instituted. At our institution, family members were counseled meticulously on the importance for strict compliance with post-operative follow up schedule. 


\section{References}

1. Gilmartin R, Bruce D, Storrs BB, Abbott R, Krach L, Ward J et al. Intrathecal baclofen for management of spastic cerebral palsy: Multicenter trial. J Child Neurol. 2000 Feb;15(2):71-7.

2. Vender JR, Hester S, Waller JL, Rekito A, Lee MR.

Identification and management of intrathecal baclofen pump complications: A comparison of pediatric and adult patients. J Neurosurg. 2006 Jan;104(1 Suppl):9-15.
3. Plassat R, Perrouin Verbe B, Menei P, Menegalli D, Mathe JF, Richard I. Treatment of spasticity with intrathecal baclofen administration: Long-term follow-up, review of 40 patients. Spinal Cord. 2004 Dec;42(12):686-93.

4. Motta F, Buonaguro V, Stignani C. The use of intrathecal baclofen pump implants in children and adolescents: Safety and complications in 200 consecutive cases. J Neurosurg. 2007 Jul;107(1 Suppl):32-5.
5. Follett KA, Boortz-Marx RL, Drake JM, DuPen S, Schneider SJ, Turner MS, et al. Prevention and management of intrathecal drug delivery and spinal cord stimulation system infec tions. Anesthesiology. 2004 Jun;100(6):1582-94.

6. Deer T, Chapple I, Classen A, Javery K, Stoker V, Tonder L, et al. Intrathecal drug delivery for treatment of chronic low back pain: Report from the national outcomes registry for low back pain. Pain Med. 2004 Mar;5(1):6-13.

\section{Brain power}

\author{
Jefferson. \\ Hospital for Neuroscience
}

1-800-JEFF-NOW

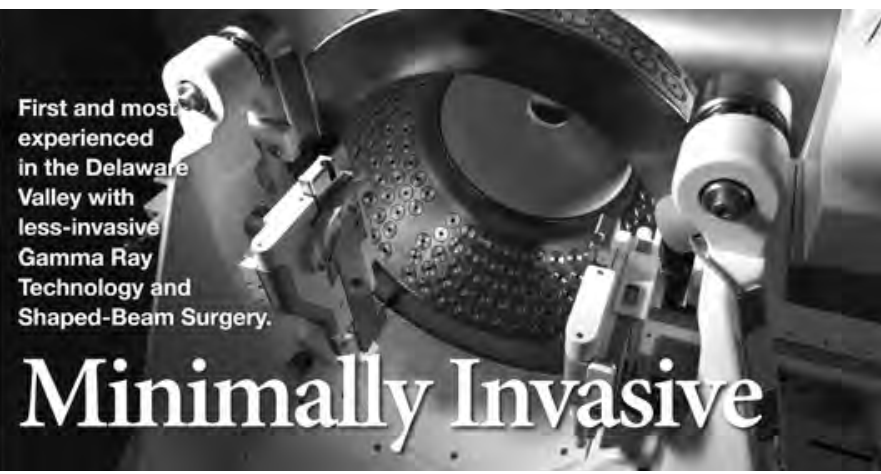

